\title{
The use of cone beam computed tomography in establishing the etiology of an impacted tooth
}

SADJ February 2020, Vol. 75 No. 1 p37 - p42

E Thomas ${ }^{1}$, MPS Sethusa ${ }^{2}$, R Singh ${ }^{3}$

\section{ABSTRACT}

Cone Beam Computed Tomography (CBCT), a threedimensional imaging modality, is considered a groundbreaking advance in the field of dental imaging. This case report illustrates how the use of CBCT enabled the correct diagnosis of the cause of the impaction of a central incisor in an orthodontic patient.

A 10-year-old male patient presented with the chief complaint of a clinically missing permanent upper right central incisor. After clinical examination and history taking, diagnostic radiographs such as orthopantomographs, lateral cephalograms and periapical radiographs were taken. On examination, the panoramic radiograph and lateral cephalogram demonstrated that the central incisor was impacted. The clinical signs, symptoms, and radiographic features indicated that an odontoma was causing the impaction. However, the presentation on the periapical radiographic presentation was suggestive of gemination, a developmental anomaly.

A CBCT scan was requested to confirm whether there were two structures (odontoma and impacted central) or one single structure (gemination). The CBCT scans helped in determining the correct etiology for the clinically missing central incisor and aided the clinician to arrive at the definitive diagnosis that a supernumerary tooth was responsible for the impaction. The scan also helped in identifying the optimal path for surgical removal of the supernumerary tooth.

Author affiliations:

1. Elizabeth Thomas: BDS (India), MSc Dent (Pead) Wits), MDS (Ortho) (India), School of Oral Health Sciences, Sefako Makgatho Health Sciences University, South Africa. ORCID Number: 0000-0003-4351-2850

2. M Peter S Sethusa: $B$ Rad (Diagn) (Medunsa), BDS (Medunsa), PG Dip in Dent (Stell), M Dent (Ortho (UL), PG Dip (UCT), School of Ora Health Sciences, Sefako Makgatho Health Sciences University, South Africa.

ORCID Number: 0000-0002-0884-3008

3. Rajbaran Singh: BChD (UWC), Dip HRM (BMTCSA), MSc (UP), School of Oral Health Sciences, Sefako Makgatho Health Sciences University, South Africa.

Corresponding author: Elizabeth Thomas

School of Oral Health Sciences, Sefako Makgatho Health Sciences University, South Africa.

Mailing address: Postnet Suite 1007, Private Bag X9, Benmore 2010

Email: lizathomas67@gmail.com

Author contributions:

1. Elizabeth Thomas: Principal researcher $-75 \%$

2. M Peter S Sethusa: Writing article $-20 \%$

3. Rajbaran Singh: Advisor - $5 \%$

\section{ACRONYMS}

ALARA Principle: As Low As Reasonably Achievable CBCT: $\quad$ Cone Beam Computed Tomography

FOV: Field of Vision

\section{INTRODUCTION}

Disruptions to normal development may lead to the apparent absence of a tooth or teeth from the dental arcade. Several reasons are discussed in the literature for "missing" teeth, including tooth agenesis, supernumerary teeth, tooth malformations or dilacerations, cysts or pathological entities such as odontomas in the eruption pathway, retained primary incisors, inadequate space in the arch and syndromic conditions. ${ }^{1,2}$

When there are several possible etiologies for a problem, determining the correct diagnosis can be challenging. Generating a differential diagnosis whereby one develops a list of possible conditions is an important step in management.

Clinical reasoning and diagnostic aids such as radiographs help to rule out some possibilities and contribute to the conclusion of a final diagnosis. In order to arrive at an accurate diagnosis and correct treatment plan, practitioners may rely on findings from imaging diagnostic aids such as orthopantomographs, peri-apicals and lateral cephalograms. ${ }^{3}$

Conventional radiographs are essentially two-dimensional (2D) representations of three-dimensional (3D) objects. These 2D images suffer from limitations such as image enlargement and structure overlap, leading to a wide differential diagnosis. Often, information gleaned from two-dimensional radiographs is insufficient and misleading, especially in cases where structures may overlap such as in supernumerary or impacted teeth. ${ }^{4}$

Two dimensional radiological presentations of dental anomalies and odontogenic tumours characterized by the presence of mineralized dental tissues sometimes present a diagnostic dilemma. ${ }^{5}$ Cone beam computed tomography $(\mathrm{CBCT})$ has emerged as a viable $3 \mathrm{D}$ imaging modality, providing a unique perspective in orthodontic diagnosis and treatment planning of clinically missing/ impacted teeth. Images can be viewed in coronal, sagittal, oblique sections and various planes, thus offering optimal viewing and avoiding superimpositions. ${ }^{6}$ 
This case report highlights the superiority of CBCT images compared with conventional two-dimensional radiographs in the diagnosis of the etiology of an impacted central incisor.

In the present case, the findings of the panoramic radiograph and the lateral cephalogram suggested the presence of an odontoma while the periapical view indicated the developmental anomaly of gemination.

CBCT, however, revealed a supernumerary tooth lying in close proximity to the crown of the impacted central incisor tooth. CBCT narrowed the wide differential diagnosis for the etiology of a clinically missing upper right central incisor in this orthodontic patient to a supernumerary tooth.

\section{CASE REPORT}

A 10-year-old male patient presented at the Department of Orthodontics, Sefako Makgatho Health Sciences University, South Africa, with the chief complaint of a missing upper right permanent central incisor. The medical history revealed that he was a healthy child.

The dental history reported that the upper right permanent central incisor had failed to appear, two years after the contralateral permanent incisor had fully erupted into the oral cavity. Intraoral examination confirmed that the upper right permanent central incisor was clinically missing.

The panoramic radiograph (Figure 1) revealed an impacted upper right permanent central incisor with a mass having the radiological density of a tooth-like structure that appeared to be superimposed at the incisal third of the permanent incisor tooth. Taking into account the dental history, clinical signs, symptoms and radiological features a diagnosis of "odontoma" was considered. ${ }^{7}$
The periapical radiographs (Figures 2 and 3) of the patient demonstrated an unerupted right central incisor showing features of coronal gemination. The incisor tooth displayed altered shapes of hard tissue and pulp.

Radiopaque enamel outlines and coronal invaginations were seen. There was a single pulpal chamber. Since the total count of teeth, including the impacted central, was normal, a diagnosis of gemination was made.
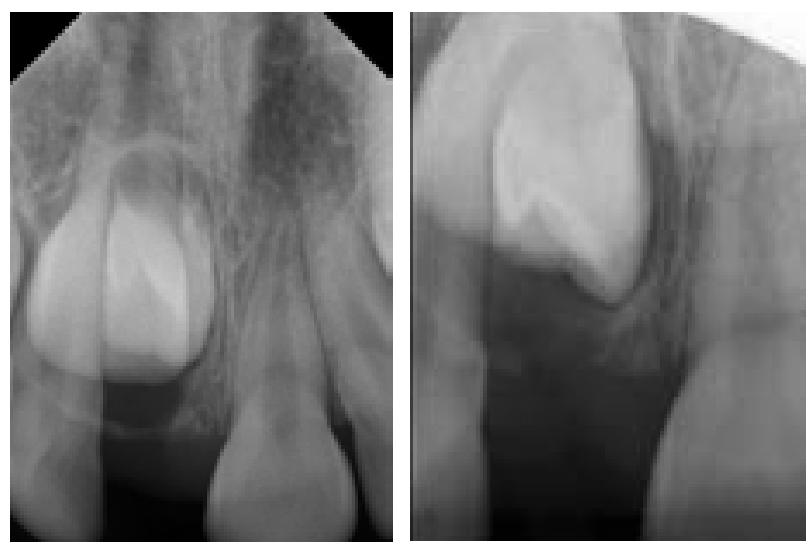

Figure 2 and 3. Periapical views of the central incisor region.

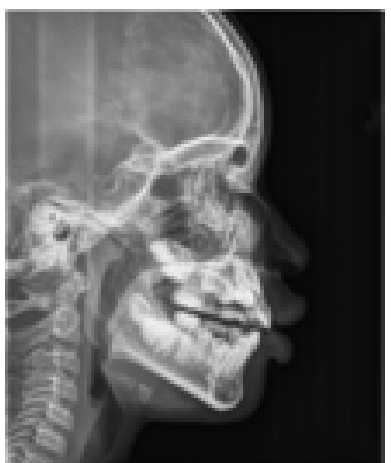

Figure 4. Lateral Cephalogram.

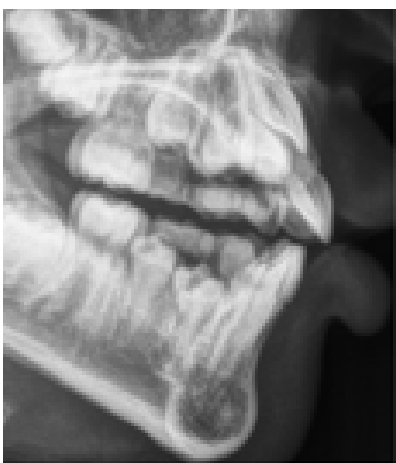

Figure 5. Magnified view of dentition.

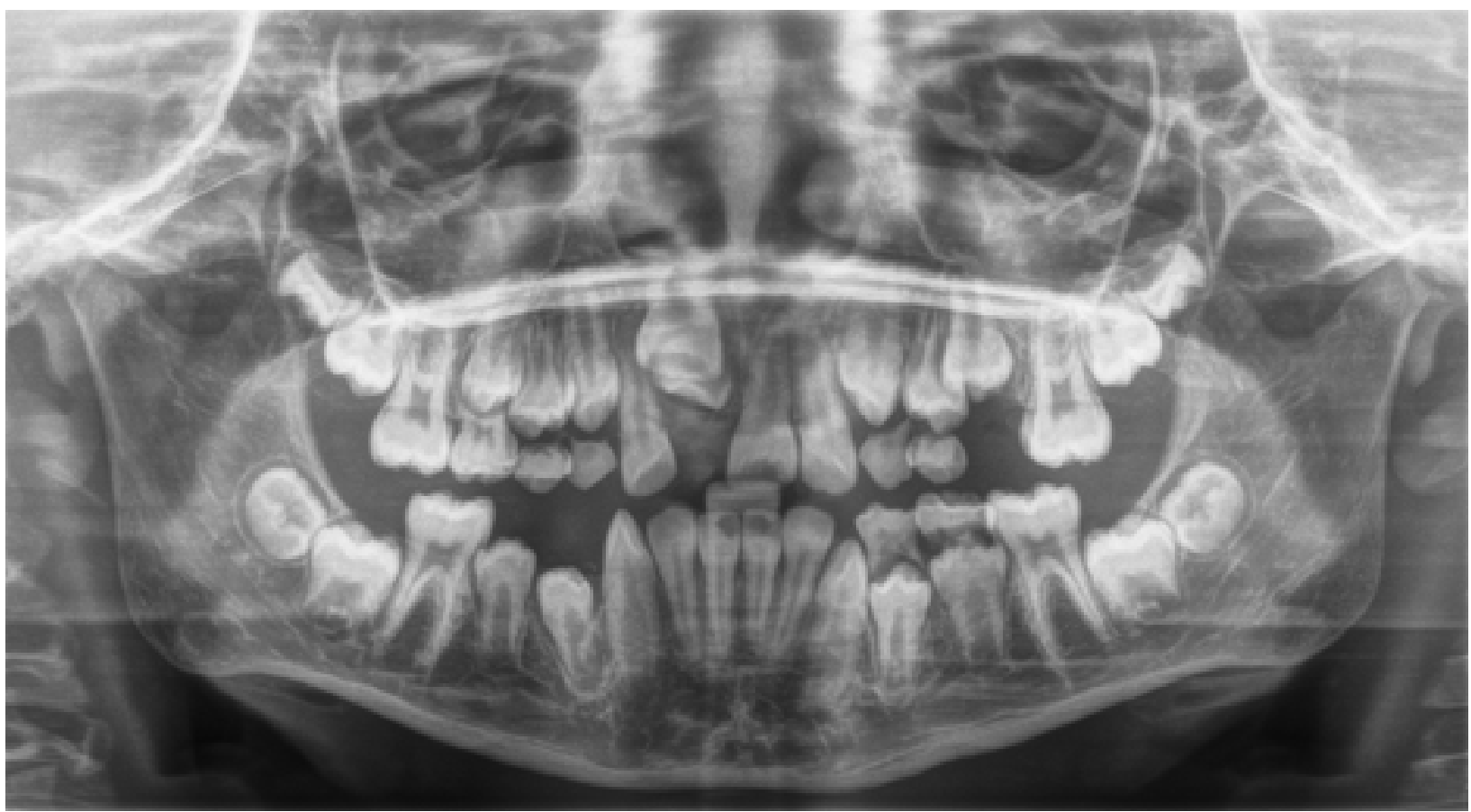

Figure 1. Panoramic radiograph. Note the mass overlying the incisal third of the crown of the 11 . 
Examination of a lateral cephalogram (Figures 4 and 5) demonstrated, on the lingual aspect of the impacted tooth, a radio-dense bell-shaped structure with invaginations and a central radiopaque spherical mass. It was not clear whether it was attached to the tooth or not.

CBCT imaging was requested to identify whether there were two structures (odontoma and impacted central) or one entity (gemination).

Radiological investigation was performed with a CBCT utilizing the following protocol: $6 \mathrm{~cm} \times 6 \mathrm{~cm}$ field of view (FOV), $110 \mathrm{kV}, 17.73 \mathrm{~mA}, 5.4$ seconds exposure time and $0.150 \mathrm{~mm}$ slice thickness. Axial, coronal and sagittal images were evaluated.

These CBCT sections (Figures 6, 7 and 8) demonstrated the appearance of a single, unilateral supernumerary tooth infero-palatal to an impacted right central incisor that had been displaced labially and superiorly. Both the supernumerary tooth and the impacted tooth were surrounded by individual pericoronal follicular spaces.
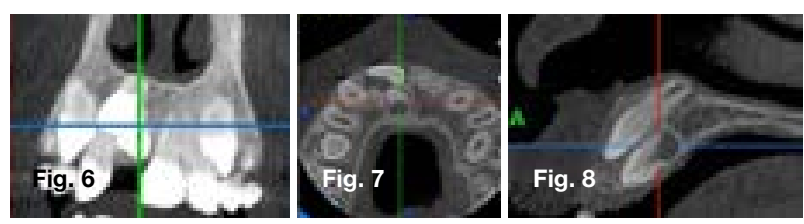

Figure 6. Axial image.

Figure 7. Coronal image.

Figure 8. Sagittal image.

CBCT proved that the differential diagnoses derived from conventional radiographs were inaccurate. It was actually a supernumerary tooth that was impeding the eruption of the right permanent central incisor.

The final diagnosis for the cause of the delayed eruption of the upper right permanent central was confirmed as a supernumerary tooth of the tuberculate variety after surgical intervention (Figures 9, 10 and 11). ${ }^{9}$

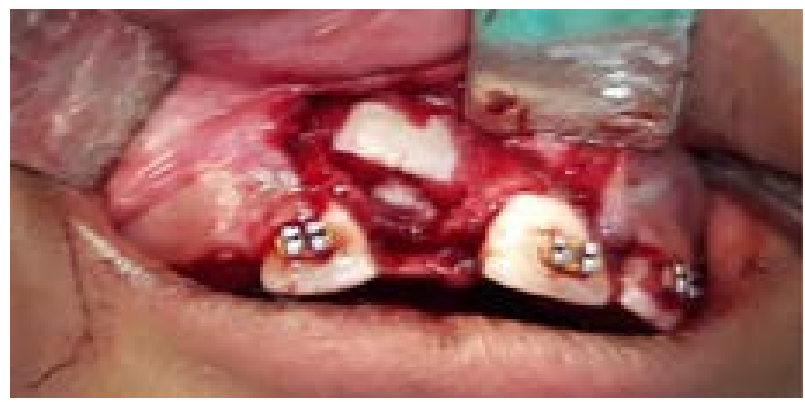

Figure 9. Surgical exposure of central incisor and supernumerary tooth.
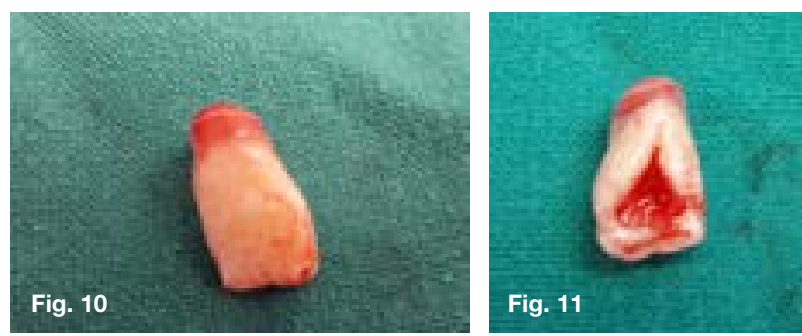

Figure 10. Labial aspect of extracted supernumerary.

Figure 11. Lingual aspect.

\section{DISCUSSION}

A correct diagnosis is the key to finalizing a correct treatment plan in the management of such cases. Accurate imaging and visualization are central to diagnostic and treatment planning processes.

Imaging techniques routinely used for diagnostic purposes in orthodontics include orthopantomographs and lateral cephalograms. The orthopantomograph enables the orthodontist to have a general overview of the dentition and supporting structures and helps to pinpoint anomalies or pathologies occurring in this region.

Although orthopantomographs are invaluable diagnostic tools, areas or structures outside the focal trough are prone to distortion. Other problems commonly encountered are magnification or overlapping of objects. The definition of structures in the panoramic $x$-ray is not as sharp as that seen on periapical or bitewing radiographs. As a result of these deficiencies, additional views may be required for patients presenting with a pathology in the incisor region. ${ }^{10,11}$

Lateral cephalograms are used to assess the dental, skeletal and soft tissue relations of an orthodontic patient. Lateral cephalometric analysis has been used widely in orthodontics as an investigative technique to evaluate growth and treatment responses. ${ }^{6}$ The cephalogram also helps in the identification of anomalies or pathologies present in the jaws as well as impacted teeth. However, there are several disadvantages.

Cephalometric analyses are based on the expectation of excellent superimposition of the left and right sides at the mid-sagittal plane. Such perfect superimpositions are rarely seen due to slight facial asymmetry in individuals as well as faulty head positioning. Major errors are also encountered due to the ambiguity in locating anatomical landmarks due to poorly defined outlines and shadows. ${ }^{12}$

Periapical views of upper and lower teeth are usually taken in orthodontics to determine the root lengths, shape and form. A review of the literature shows that these features play a role in determining the susceptibility of incisors to root resorption. ${ }^{13}$ Knowledge of these factors before commencing treatment helps the orthodontist to be prudent with the application of forces.

Therefore, periapical radiographs of the incisors of orthodontic patients are recommended before commencing treatment. Periapical views, however, are limited in terms of the possibility of an overlapping of the images of buccally or lingually impacted teeth, leading to incorrect interpretations. ${ }^{14}$

Imaging is a valuable diagnostic addition to the clinical assessment of an orthodontic patient. However, intraoral imaging procedures such as periapical views and extra-oral procedures including panoramic $\mathrm{x}$-rays and lateral cephalograms used individually or in combination suffer from the same inherent limitations of all two dimensional projections, namely magnification, distortion, superimposition and misrepresentation of structures. ${ }^{15}$ 
In this particular case, interpretation of all these twodimensional conventional radiographs failed to produce a definitive diagnosis. The differential diagnosis varied from "odontoma," a benign tumour, to "gemination," a developmental anomaly.

The World Health Organization (WHO) classifies odontomas within the category of odontogenic tumours that are composed of epithelial and mesenchymal components of the dental apparatus with or without the formation of mineralized dental tissues.

These lesions are considered as hamartomas and not as true neoplasms. ${ }^{16,17}$ Typically, they are divided into compound and complex odontomas. ${ }^{18}$ Complex odontomas are less common than compound odontomas. A dilated odontoma has been described as yet another type of odontoma. ${ }^{16}$ This is a single structure that may be a more severe form of dens in dente.

Odontomas are the most common type of odontogenic tumours and are generally painless and asymptomatic. The majority of odontomas appear before the age of 20 , hence are discovered during childhood or adolescence. They are seen more frequently in the maxillary anterior region than in the mandible and are often associated with unerupted permanent teeth. ${ }^{19}$ The canines, followed by upper central incisors and third molars, are the teeth most frequently impacted by odontomas. ${ }^{20}$

Odontomas develop and mature while the neighbouring teeth are forming and conclude their development once the development of the associated teeth is complete. Only rarely does an odontoma erupt into the oral cavity. If associated with an impacted tooth, odontomas are usually removed by surgical excision. They do not recur and after their removal, the impacted tooth usually erupts into the oral cavity on its own or otherwise may require orthodontic traction.

Radiologically, three stages can be seen in the development of an odontoma. In the first stage, the tumour appears radiolucent due to lack of calcification of dental tissues. In the intermediate stage, a partial calcification is noted and in the final stage the odontoma appears radiopaque with a radiolucent halo. ${ }^{21}$ Compound odontomas are seen as calcified structures resembling teeth within a well-defined radiolucent lesion. ${ }^{5,7}$

A complex odontoma presents as a more or less amorphous mass with the radio-density of a tooth structure, but bearing no anatomical resemblance to a tooth, and is surrounded by a thin radiolucent area with a smooth periphery. ${ }^{5}$ In some cases, the calcified matrix is predominant while in others there are islands of pulp tissue along with cords and buds. ${ }^{21}$

The dilated odontome is considered the most severe form of dens invaginatus or dens in dente. The former is characterized by the invagination or in folding of enamel surface into the interior of a tooth. ${ }^{22}$ The invagination can occur either at the incisal edge or at the cingulum area. It is seen most frequently affecting the permanent maxillary lateral incisors followed by maxillary central incisors, premolars and canines. They are very rarely observed in the posterior region. In its most affected form, the tooth is severely malformed. Radiologically, it presents with a circular or oval form and a radiolucent interior. ${ }^{16}$

A differential diagnosis, generated from assessment of the periapical X ray, was that of "gemination". This dental anomaly occurs when a single tooth bud attempts to divide. This may result in the invagination of the crown with partial division. It can occur in both primary and permanent dentitions and is usually seen in the incisor region. It is commonly detected after the abnormal tooth erupts into the oral cavity.

Radiographic evaluation reveals alteration in the normal contour of the hard tissue - namely, clefts and invaginations in the enamel outlines and the pulp chamber is usually single and enlarged. ${ }^{16}$ A geminated tooth in the anterior region may produce compromised esthetics. ${ }^{23}$

In addition, areas of invaginations are prone to caries and sometimes result in pulpal inflammation. In treatment, the crown may be reshaped or restored or left untreated with periodic reviews if esthetics is not a concern of the patient. ${ }^{16}$

In this case, traditional two-dimensional radiographs used as diagnostic aids, failed to provide a definitive, accurate diagnosis. Studies have shown that CBCT scans are useful in refining diagnoses and thus contribute significantly to a higher confidence level in a the diagnostic acumen of the clinician. ${ }^{24,25} \mathrm{~A}$ correct diagnosis leads to a successful treatment plan and a gratified, contented patient.

Clinicians may request additional 3D images using CBCT if conventional radiographs fail to provide enough information for diagnosis, surgical intervention and orthodontic therapy. ${ }^{14}$ Since CBCT has higher radiation doses, the basic principles to which an orthodontist should adhere before requesting cone beam computed tomography are:

1. CBCT examinations should not be used routinely and indiscriminately for all patients

2. A history and clinical examination must be conducted before doing a CBCT assessment.

3. CBCT assessments must be justified for each patient. CBCT images must provide new information when compared with that coming from conventional radiographs

4. CBCT field of view (FOV) should be controlled as much as possible. This helps to reduce the radiation dose and achieve the ALARA principle (As Low As Reasonably Achievable). ${ }^{14}$

In the present case, a CBCT scan revealed that it was a supernumerary tooth that was causing the impaction of the central incisor. Supernumerary teeth denote those formed in excess of the number found in normal series and are more prevalent in the permanent dentition. Most frequently found in the central incisor region, they might be associated with delayed eruption of the adjacent incisors. ${ }^{2,26,27}$ 
Supernumerary teeth in the permanent dentition are classified according to their position as mesiodens, paramolar or distomolar. Mesiodens are typically found between the maxillary central incisors. Mesiodens may be single or multiple, unilateral or bilateral, erupted or unerupted. Their orientation could be vertical, horizontal or inverted. Paramolar is a supernumerary molar found buccally or lingually in relation to the maxillary molars or in the buccal interproximal space between the second and third molars. Distomolar is a small and rudimentary molar located distal to the third molar. ${ }^{26,28}$

Supernumerary teeth are classified according to their morphology as conical, tuberculate, supplemental or odontoma. ${ }^{26,28}$ Conical supernumerary teeth are small peg-shaped teeth with root formation ahead of or corresponding to that of permanent incisors. It is the most common supernumerary tooth in the permanent dentition. It is termed as a mesiodens and is present between upper central incisors and occasionally may be found high in the palate in an inverted or horizontal position.

Tuberculate teeth are supernumerary teeth, which are larger than the conical type and have more than one cusp or tubercle. They are short, barrel-shaped teeth with normal appearing crown and a rudimentary root. Root formation is delayed when compared with the permanent incisors. They are characteristically located on the palatal aspect of the permanent central incisors, are frequently associated with delayed eruption of the incisors and rarely erupt into the oral cavity. ${ }^{1}$

Supplemental teeth refers to duplication of teeth in the normal series and are found at the end of a tooth series. In the permanent dentition, they appear most commonly as extra maxillary and mandibular lateral incisors. Odontomas have no regular shape. This last category is not universally accepted as a supernumerary, however. ${ }^{28,29}$

Similarities concerning their topographic distribution, clinical features and pathologic manifestations between odontomas and supernumerary teeth have been quoted. ${ }^{17}$ There appears to be some possibility of common genetic and immune-histochemical etiologic factors for these conditions. From a nosological point of view, odontomas and supernumeraries are classified as separate entities, but seem to be an expression of a similar process that is either malformative or hamartomatous.

Most supernumerary teeth are located in the anterior maxillary region. ${ }^{2,30}$ They may remain asymptomatic without any clinical manifestations. ${ }^{27}$ Sometimes malocclusions caused by supernumerary teeth, such as impacted teeth, crowding or diastema, particularly in the anterior region, may negatively affect the esthetics, mastication and speech of children, who might then become objects of ridicule in their peer groups, affecting their psychological development. Early detection, supervision and management of supernumerary teeth is crucial in avoiding these problems. ${ }^{31}$

Detection of supernumerary teeth is best achieved by a thorough clinical and radiographic examination.
The application of conventional radiographic techniques together with $\mathrm{CBCT}$ in order to determine the position of supernumerary and impacted teeth has been studied previously. ${ }^{32,33}$

The results show that both techniques are useful as initial diagnostic tools but the CBCT images provide more detailed information about the position of the teeth, the exact dimension of supernumerary and impacted teeth and the presence or absence of root resorption. ${ }^{25,33}$ A frequent indication for CBCT in orthodontics is for eruptive disorders like impacted teeth and supernumerary teeth. ${ }^{14,34}$ By using 3D visualization, CBCT provides information in all three planes and thus enhances diagnostic accuracy. This is an important attribute when contemplating surgical removal of supernumerary teeth which have resulted in impaction of neighboring permanent teeth. The need for accurate diagnostic 3D information also becomes apparent when considering the proximity of supernumerary teeth located in the anterior maxillary region to neighboring teeth and vital structures like the nasopalatine canal, nasal floor or the floor of the mouth. ${ }^{35}$

\section{CONCLUSION}

It is recommended that in the case of eruptive disorders, including impacted teeth, it is advisable to investigate the etiology of the condition by detailed history taking, clinical examination and the critical assessment of diagnostic aids like radiographs. If conventional two-dimensional radiographs fail to provide a definitive diagnosis, then the use of CBCT scans is recommended.

CBCT scans should be used only when the potential benefits for diagnosis and treatment planning outweigh the potential risks of an increased radiation dose. In the present case, CBCT imaging led to a change in diagnosis and treatment planning and was justified. CBCT imaging also assisted the surgeon in determining the least invasive point of entry for removal of the supernumerary tooth.

\section{Acknowledgements}

The technical support provided by Mxamli BE, Radiographer in the Department of Maxillofacial Radiology is gratefully acknowledged.

\section{References}

1. Manuja N, Nagpal R, Singh M, Chaudhary S. Management of delayed eruption of permanent maxillary incisor associated with the presence of supernumerary teeth: a case report. Das UM, editor. Int J Clin Pediatr Dent. 2011; 4: 255-9.

2. Chou S-T, Chang H-P, Yang Y-H, Lung C-Y, Tseng Y-C, Pan $\mathrm{C}-\mathrm{Y}$, et al. Characteristics of supernumerary teeth among nonsyndromic dental patients. J Dent Sci [Internet]. 2015; 10(2): 133-8.

3. Bruks A. Radiographic examinations as an aid to orthodontic diagnosis and treatment planning. Swed Dent J. 1999; 23(2-3): 77-85.

4. Agrawal JM, Agrawal MS, Nanjannawar LG, Parushetti AD. CBCT in orthodontics: the wave of future. $J$ Contemp Dent Pr. 2013; 14(1): 153-7. 
5. Barba LT, Campos DM, Rascón MMN, Barrera VAR, Rascón AN. Descriptive aspects of odontoma: literature review. Rev Odontológica Mex [Internet]. 2016; 20(4): 265-9.

6. Karatas $\mathrm{OH}$, Toy $\mathrm{E}$. Three-dimensional imaging techniques: A literature review. Eur J Dent. 2014; 8(1): 132.

7. de Oliveira BH, Campos V, Marçal S. Compound odontoma--diagnosis and treatment: three case reports. Pediatr Dent [Internet]. 2001; 23(2): 151-7.

8. Le Gall M, Philip C, Aboudharam G. Orthodontic treatment of bilateral geminated maxillary permanent incisors. Am J Orthod Dentofac Orthop. 2011; 139(5): 698-703.

9. Sagarika Shukla, Ashi Chug KIA. Role of cone beam computed tomography in diagnosis and treatment planning in Dentistry: an update. J Int Soc Prev and Community Dent. 2017; 7(3): S125-136

10. Anthonappa RP, King NM, Rabie ABM, Mallineni SK. Reliability of panoramic radiographs for identifying supernumerary teeth in children. Int J Paediatr Dent [Internet]. 2012; 22(1): 37-43.

11. Haney E, Gansky SA, Lee JS, Johnson E, Maki K, Miller AJ, et al. Comparative analysis of traditional radiographs and cone-beam computed tomography volumetric images in the diagnosis and treatment planning of maxillary impacted canines. Am J Orthod Dentofac Orthop [Internet]. 2010; 137(5): 590-7.

12. Speidel TM. Orthodontic cephalometry [Internet]. Am J Orthod Dentofac Orthop. 1997; 111: 458-9.

13. Sameshima GT, Asgarifar KO. Assessment of root resorption and root shape: periapical vs panoramic films. Angle Orthod. 2001; 71(3): 185-9.

14. Garib DG, Calil LR, Leal CR, Janson G. Is there a consensus for CBCT use in Orthodontics? Dental Press J Orthod [Internet]. 2014; 19(5): 136-49.

15. Scarfe WC, Farman AG. What is Cone-Beam CT and how does it work? Dent Clin North Am. 2008; 52(4): 707-30.

16. White SC, Pharoah MJ. Radiographic interpretation. In: Oral Radiology: Principles and Interpretation. $6^{\text {th }}$ ed. St. Louis, MO: Mosby Elsevier; 2009: 378-80.

17. Pippi R. Odontomas and supernumerary teeth: Is there a common origin? Int J Med Sci. 2014; 11(12): 1282-97.

18. Gedik R, Müftüoglu S. Compound odontoma: differential diagnosis and review of the literature. West Indian Med J. 2014; 63(7): 793-5

19. Soluk Tekkesin M, Pehlivan SOV. Clinical and histopathological investigation of odontomas: review of the literature and presentation of 160 cases. J Oral Maxillofac Surg. 2012; 70: 1358-61.

20. Katz RW. An analysis of compound and complex odontomas. ASDC J Dent Child. 1989; 56(6): 445-9.

21. Philipsen HP, Reichart PA. Mixed odontogenic tumours and odontomas. Considerations on interrelationship. Review of the literature and presentation of 134 new cases of odontomas. Oral Oncol. 1997; 33(2): 86-99.

22. Dineshshankar J, Sivaraman S, Yasmeenahamed S. Dens Invaginatus: History, etiology, classification, clinical features, radiographic feature, histological findings and management. Saudi J Oral Dent Res. 2016; 1(3): 151-5.

23. Steinbock N, Wigler R, Kaufman AY, Lin S, Abu-El Naaj I, Aizenbud D. Fusion of central incisors with supernumerary teeth: A 10-year follow-up of multidisciplinary treatment. J Endod [Internet]. 2014; 40(7): 1020-4.

24. Kapila SD, Nervina JM. CBCT in orthodontics: Assessment of treatment outcomes and indications for its use. Dentomaxillofacial Radiol. 2015; 44(1): 1-19.

25. Nematolahi H, Abadi H, Mohammadzade Z, Ghadim MS. The use of cone beam computed tomography (CBCT) to determine supernumerary and impacted teeth position in pediatric patients: a case report. J Dent Res Dent Clin Dent Prospects [Internet]. 2013; 7(1): 47-50.
26. Hattab F, Yassin O, Rawashdeh M. Supernumerary teeth: Report of three cases and review of the literature. ASDC $J$ Dent Child. 1994; 61(5-6): 382-93.

27. Rajab LD, Hamdam MA. Supernumerary teeth: review of literature and a survey of 152 cases. Int J Paediatr Dent. 2002; 12: 244-54.

28. Mitchell L. An Introduction to Orthodontics. 4th ed. Oxford, Uk: Oxford University Press; 2013: 24-25.

29. Garvey MT, Barry HJ BM. Supernumerary teeth-an overview of classification, diagnosis and management. J Can Dent Assoc. 1999; 65: 612-6.

30. McBeain M, Miloro M. Characteristics of supernumerary teeth in nonsyndromic population in an urban dental school setting. J Oral Maxillofac Surg [Internet]. 2018; 76(5): 933-8.

31. Tokarska P. Surgical treatment procedures based on material supplied by the Department of Orthodontics of the Medical University of Warsaw. Mezjodens - czestosc wystepowania, powikłania i metody wczesnego leczenia na podstawie dokumentacji Zakładu Ortodoncji WUM Zakład Ortodoncji, W. 2015; (November).

32. Hidalgo-Rivas JA, Theodorakou C, Carmichael F, Murray B, Payne M, Horner K. Use of cone beam CT in children and young people in three United Kingdom dental hospitals. Int $\mathrm{J}$ Paediatr Dent. 2014; 24: 336-48.

33. Haney E, Gansky SA, Lee JS, Johnson E, Maki K, Miller AJ, et al. Comparative analysis of traditional radiographs and cone-beam computed tomography volumetric images in the diagnosis and treatment planning of maxillary impacted canines. Am J Orthod Dentofac Orthop [Internet]2014; 137(5): 590-7.

34. Mossaz J, Kloukos D, Pandis N, Suter VGA, Katsaros C, Bornstein MM. Morphologic characteristics, location, and associated complications of maxillary and mandibular supernumerary teeth as evaluated using cone beam computed tomography. Eur J Orthod. 2014; 36(6): 708-18.

35. Becker A, Chaushu S, Casap-Caspi N. Cone-beam computed tomography and the orthosurgical management of impacted teeth. J Am Dent Assoc [Internet]. 2010; 141: 14-8. 